Results Preterm birth occurred in $15.6 \%$ (19/122), low birth weight (LBW) in 22.1\% (27/122) and small for gestational age fetal weight in $19.7 \%(24 / 122)$ of cases, without significant variation due to the HIV-status (only infected or Aids) or antiretroviral use.

Perinatal death occurred in 4.1\% (5/122) of the cases. The Apgar score of less than or equal to 7 in the first minute was observed in $7.4 \%(9 / 120)$ and Zidovudine intravenous in prepartum was used in $92.5 \%(110 / 120)$ of cases. After a followup of more than 18 months of post $\neg$ natal life, vertical transmission (TV) was verified in 6 cases $(4.9 \% ; 6 / 122)$.

Conclusion LBW, small for gestational age fetal weight, preterm birth and perinatal death occurrences were higher than the expected values in this research, indicating the need for preventive actions.

Disclosure No significant relationships.

\section{P212 PSYCHOSOCIAL FACTORS AFFECTING THE CAREGIVERS OF PEDIATRIC HIV PATIENTS IN RETURNING FOR TREATMENT}

${ }^{1}$ Kelsey Buehler*, ${ }^{2}$ Milton Rahman, ${ }^{2}$ Eiman Mahmoud. ${ }^{1}$ Touro University, 3rd Year Medical

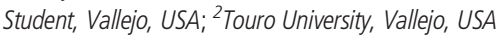

10.1136/sextrans-2019-sti.359

Background Stringent HIV treatment adherence is paramount for expanding life and decreasing mortality (Adefolalu \& Nkosi, 2013). However, pediatric adherence to therapy is a complex multifactorial issue that is affected by many issues such as characteristics of the child, caregiver(s), family, regimen, and culture (Haberer and Mellins, 2009). This study aims to explore the psychosocial influences on adherence through examining patient and caregiver return to clinic for treatment. The psychosocial influences we plan to investigate can be divided into the following: Stigma, women's empowerment, socioeconomic factors, stress, and family characteristics.

Methods 67 caregivers of pediatric HIV patients from Shirati Hospital in Tanzania were given quantitative surveys regarding psychosocial barriers to returning the patient for HIV treatment. Survey questions included topics about income, primary caregivers, stigma about HIV, average number of people known with HIV, transportation, stress levels, missed HIV appointments and skipped medication treatments.

Results Data was split into adherent and non-adherent groups. Non-adherent group missed either an appointment or medication dosage for the patient in the past 6 months (G1). Adherent group had not missed either (G2). Percentage male patient: G1: 63.6\%; G2: 51.1\% Average caregiver age: G1: 39.5 years; G2: 36.5 years Caregiver sex: G1: 41.7\% male; G2: $19.2 \%$ male Caregiver relationship to patient: G1 $41.7 \%$ mother; G2: $56.6 \%$ mother Person taking patient to clinic is primary caregiver: G1: 63.6\%; G2: $80 \%$ Patient has more than one caregiver: G1 41.7\%; G2: 52.7\% Caregiver at clinic believes HIV is shameful: G1: 9\%; G2: $31.5 \%$ Easily able to get clinic: G1: 25\%; G2: 49\% Perceived stress levels: G1: 3.37, G2: 2.96 (1-5 scale)

Conclusion Summary of unique findings include the adherent group displaying increased shameful preceptions of HIV. The non-adherent group had an increased average caregiver age and were more likley to be caring for a male patient.
Disclosure No significant relationships.

\section{P216 GENDER-BASED VIOLENCE AND THE ASSOCIATED PSYCHOSOCIAL AND MENTAL HEALTH ISSUES AMONG FILIPINO HIV-POSITIVES}

Evangeline Castronuevo-Ruga*. De La Salle University-Dasmarinas, Psychology Department, Dasmarinsa City, Cavite, Philippines

\subsection{6/sextrans-2019-sti.360}

Background Gender-based violence (GBV) has generated practitioners' and researchers' interest since the surge of the women's movement few decades ago in the Philippines. Meanwhile, HIV-AIDS gained similar attention with the disclosure of the first ever case of the country in mid-80s. Only recently, however, has the intersectionality of these two phenomena been looked into by the research community elsewhere and has yet to be systematically studied locally. This research, therefore, attempts to map out the lived experiences of People Living with HIV (PLHIV) who have undergone gender-based violence (GBV) and looks into the consequent psychosocial and mental health issues.

Methods We used qualitative design to describe and understand the participants' gender-based violence (GBV) experiences relative to their becoming HIV-positives. Data was generated by a partner health non-government organization (NGO), the Action for Health Initiatives, (Achieve, Inc.), by recruiting 24 participants from the highly vulnerable groups of HIV positives, namely: entertainers/sex workers; men having sex with men (MSM); overseas Filipino workers (OFWs), and injecting drug users (IDUs) who were initially part of a survey done on a related study.

Using focus group discussion and in depth interviews, the participants were asked about the gender-based violence that they experienced early in life, about their experience with the Human Immuno-Deficiency virus (HIV), and about the psychosocial impact of these in their lives. In agreement with the partner NGO, the authors did the thematic analysis on the accounts generated.

Results Thematic analysis reveals that the participants experienced various forms of gender-based violence, e.g., sexual, emotional/psychological, economic, verbal, physical; and expressions of stigma and discrimination, which in turn, led to manifestations of psychosocial and mental health issues like trauma, depression, internalized homophobia, greater health risks and risk-taking behaviors, among others.

Conclusion Worth considering is the possibility that the consequent risk-taking and self-injurious tendencies played a role in their eventual contraction of HIV.

Disclosure No significant relationships.

\section{P217 'STIGMA AND LEVEL OF CARE AMONG HEALTH CARE PROVIDERS TO HIV/AIDS PATIENTS'}

Felisse Julien Jegonia*. Maria Reyna-Xavier University Hospital, Department of Internal Medicine, Cagayan De Oro City, Philippines

10.1136/sextrans-2019-sti.361 